\title{
Re-evaluating reaction rates relevant to nova nucleosynthesis from a nuclear structure perspective
}

\author{
1 Department of Physics, University of York, York YO10 5DD, UK \\ 2 Physics Division, Argonne National Laboratory, Argonne, IL 60439, USA \\ 3 School of Physics, University of Edinburgh, Edinburgh, UK \\ 4 Department of Physics, University of Jyväskylä, Jyväskylä, Finland \\ 5 ICREA, E-08010 Barcelona, Spain \\ 6 Institut d'Estudis Espacials de Catalunya (IEEC), E-08034, Barcelona, Spain
}

D.G. Jenkins ${ }^{1, a}$, C.J. Lister ${ }^{2}$, R.V.F. Janssens ${ }^{2}$, T.L. Khoo ${ }^{2}$, E.F. Moore ${ }^{2}$, K.E. Rehm ${ }^{2}$, D. Seweryniak ${ }^{2}$ A.H. Wuosmaa ${ }^{2}$, T. Davinson ${ }^{3}$, P.J. Woods ${ }^{3}$, A. Jokinen ${ }^{4}$, H. Penttila ${ }^{4}$, G. Martínez-Pinedo ${ }^{5,6}$, and J. Jose ${ }^{6}$

Received: 24 June 2005 /

Published online: 24 February 2006 - (C) Società Italiana di Fisica / Springer-Verlag 2006

\begin{abstract}
Conventionally, reaction rates relevant to nova nucleosynthesis are determined by performing the relevant proton capture reactions directly for stable species, or as has become possible more recently in inverse kinematics using short-lived accelerated radioactive beams with recoil separators. A secondary approach is to compile information on the properties of levels in the Gamow window using transfer reactions. We present a complementary technique where the states of interest are populated in a heavy-ion fusion reaction and their gamma decay studied with a state-of-the-art array of high-purity germanium detectors. The advantages of this approach, including the ability to determine resonance energies with high precision and the possibility of determining spins and parities from gamma-ray angular distributions are discussed. Two specific examples related to the ${ }^{22} \mathrm{Na}(\mathrm{p}, \gamma)$ and ${ }^{30} \mathrm{P}(\mathrm{p}, \gamma)$ reactions are presented.
\end{abstract}

PACS. $26.30 .+\mathrm{k}$ Nucleosynthesis in novae, supernovae, and other explosive environments -21.10 . Tg Lifetimes $-27.30 .+\mathrm{t} 20 \leq A \leq 38$

\section{Introduction}

Two key issues relating to nova nucleosynthesis have been recently identified. The former pertains to the probability of making direct observation of nova explosions through the detection of gamma rays following the beta decay of certain nuclear species produced in the explosion such as ${ }^{22} \mathrm{Na}$ and ${ }^{26} \mathrm{Al}[1]$. In this respect, ${ }^{22} \mathrm{Na}$ which decays, with a $2.602 \mathrm{y}$ half-life, into a short-lived excited state of ${ }^{22} \mathrm{Ne}$, emitting a $1.275 \mathrm{MeV} \gamma$-ray, is seen as a particularly important diagnostic of nova explosions with the expectation that explosions within a few kiloparsecs of the Sun might provide detectable $\gamma$-ray fluxes associated with ${ }^{22} \mathrm{Na}$ decay. Efforts are ongoing to detect such cosmic $\gamma$-rays, in particular, with the recently launched ESA INTEGRAL mission [2]. In order to understand the likelihood of detecting cosmic gamma rays from ${ }^{22} \mathrm{Na}$, it is important to quantify the processes responsible for the production and destruction of ${ }^{22} \mathrm{Na}$ in nova nucleosynthesis. Uncertainties in the rate of production of ${ }^{22} \mathrm{Na}$ have largely been lifted

\footnotetext{
a e-mail: dj4@york.ac.uk
}

by a study of the ${ }^{21} \mathrm{Na}(\mathrm{p}, \gamma)$ employing a radioactive ${ }^{21} \mathrm{Na}$ beam in inverse kinematics $[3]^{1}$. Prior to the work described here, there was rather more significant uncertainty in the destruction rate via the ${ }^{22} \mathrm{Na}(\mathrm{p}, \gamma)$ reaction.

A second area of inquiry is the location of the endpoint for nova nucleosynthesis. Jose has identified the ${ }^{30} \mathrm{P}(\mathrm{p}, \gamma)$ reaction as being the key determinant in this respect [5]. This reaction rate is determined on the basis of HauserFeshbach calculations as no relevant experimental measurements have been made. It is not known how reliable such a methodology might be in this case since the level density may not be high enough to make the necessary assumptions about the availability of suitable resonances in the Gamow window. Jose has shown that plausible variances in the reaction rate by a factor of 100 up or down have dramatic consequences for the endpoint of nova nucleosynthesis [5].

\footnotetext{
1 We note that some of the remaining uncertainties in this reaction have very recently been removed by a gamma-ray spectroscopy study of ${ }^{22} \mathrm{Mg}$ using the Gammasphere array [4].
} 


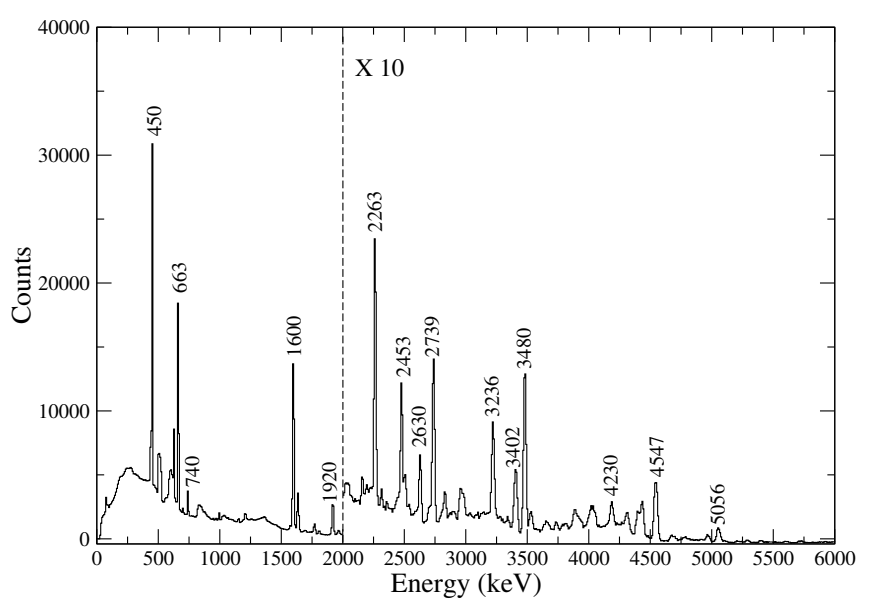

Fig. 1. Sum of double gates on the $450,1600,663$ and $2739 \mathrm{keV}$ transitions in the $\gamma-\gamma-\gamma$ cube. Strong transitions in ${ }^{23} \mathrm{Mg}$ are labelled with their energy in $\mathrm{keV}$.

\section{Example 1: ${ }^{22} \mathrm{Na}(\mathrm{p}, \gamma)$}

In the past, several methods have been employed in order to obtain the astrophysical reaction rate for the ${ }^{22} \mathrm{Na}(\mathrm{p}, \gamma)$ reaction $[6,7,8,9]$. The key to such an analysis is a detailed knowledge of properties such as the excitation energy, spin and parity of levels in the unbound region. The conventional approach to this problem is to study the ${ }^{22} \mathrm{Na}(\mathrm{p}, \gamma)$ directly by bombarding a specially prepared radioactive ${ }^{22} \mathrm{Na}$ target with protons and detecting the $\gamma$-rays following proton capture $[6,7]$. We have pursued a complementary approach in which particle-unbound states were populated in a heavy-ion fusion-evaporation reaction and their subsequent $\gamma$ decay investigated with Gammasphere, a $4 \pi$ high resolution $\gamma$-ray spectrometer consisting of 100 largevolume, high-purity germanium detectors comprising a total efficiency of around $9 \%$ for $1.33 \mathrm{MeV} \gamma$-rays [10]. This work is reported in more detail elsewhere [11].

A 10 pnA beam of ${ }^{12} \mathrm{C}$ was accelerated to $22 \mathrm{MeV}$ by the ATLAS accelerator at Argonne National Laboratory and was incident on a $40 \mu \mathrm{g} / \mathrm{cm}^{2}$ thick ${ }^{12} \mathrm{C}$ target. The resulting gamma decay was detected using the Gammasphere array. The fusion channels observed were singleproton, neutron or alpha emission leading to ${ }^{23} \mathrm{Na},{ }^{23} \mathrm{Mg}$ and ${ }^{20} \mathrm{Na}$, respectively. A $\gamma-\gamma$ matrix and a $\gamma-\gamma-\gamma$ cube were produced and analysed to obtain information on the decay schemes. The construction of the decay schemes was straightforward given the small number of residual nuclei produced and their well-known decay schemes at low excitation energies [12]. An example of the quality of the data obtained is given in fig. 1.

It is worthwhile to reflect that large $\gamma$-ray spectrometers are most commonly designed for the study of excited states of heavy nuclei where high-multiplicity cascades $(\sim 20$ photons $)$ and relatively low energies $(\sim 1 \mathrm{MeV})$ are expected. By contrast, for the astrophysical application described here, the relevant cascades have both a relatively low multiplicity and $\gamma$-ray energies which may be above $10 \mathrm{MeV}$, meaning that particular attention needs to be paid to both energy and efficiency calibrations.
In obtaining accurate $\gamma$-ray energies, we have applied a correction for the non-linearity of the array as well as the finite recoil correction for large-energy $\gamma$-rays emitted from a light nucleus. In cases where two coincident transitions were crossed over by a third transition, the corrected energy sum was compared and found to be in agreement at the $\sim 0.5-1 \mathrm{keV}$ level.

In order to assign a multipolarity to the observed transitions, a matrix was generated of $\gamma$-rays detected at all angles against those detected at $90^{\circ}$ and a matrix of all $\gamma$-rays against those detected at $32^{\circ}$ and $37^{\circ}$. The ratio $\left(R_{D C O}\right)$ of the intensities of transitions in these two matrices when gating on the "all detector" axis was extracted. This ratio was around $0.9(1)$ for pure dipole transitions and around 1.7(2) for pure quadrupole transitions. Mixed $M 1 / E 2$ dipole transitions may have various values depending on the value of the mixing ratio. As well as angular correlations, it was also possible to assign the spin/parity of states in ${ }^{23} \mathrm{Mg}$, on the basis of their similarity in both energy and decay path to analogue states of well-established spin and parity in ${ }^{23} \mathrm{Na}$ [12], for which extensive additional spectroscopic information was obtained.

The high energy of many of the $\gamma$-rays observed implies very short (femtosecond) lifetimes which are readily extracted using the fractional Doppler-shift technique [13] since it may reasonably be assumed for high-lying, unbound states that the feeding is direct. Seven matrices were sorted containing un-Doppler-corrected gamma-ray energies observed at $32^{\circ}, 50^{\circ}, 80^{\circ}, 90^{\circ}, 100^{\circ}, 130^{\circ}$ and $148^{\circ}$, respectively, against Doppler-corrected energies observed at any angle. The peak centroids were obtained for a transition at all seven angles when gated on a transition on the Doppler-corrected "all detector" axis. This was used to calculate the observed Doppler shift and, hence, the fractional Doppler shift relative to the calculated maximum Doppler shift for the given beam and target. A model prescription was used to relate the fractional Doppler shift to the lifetime of the parent state. The gamma width of the state may be deduced from the lifetime.

\subsection{Re-evaluation of the reaction rate}

The Gammasphere array affords the possibility of determining the energy of the resonances to higher accuracy than that obtainable with a spectrometer. It also allows their decay path to be observed and through angulardistribution measurements, the spin/parity of these resonances may be inferred or at least restricted to some plausible range. The $\gamma$-ray energies, angular-correlation ratios, and lifetimes of proton-unbound states in ${ }^{23} \mathrm{Mg}$ are presented in table 1 .

The resonance strengths were taken from the literature where known [14], or else were calculated from measured spectroscopic factors and calculated single-particle proton widths. The resonance strength for a state with spin, $J$, is given by

$$
\omega \gamma=\omega \frac{\Gamma_{p} \Gamma_{\gamma}}{\Gamma_{p}+\Gamma_{\gamma}},
$$


Table 1. Spectroscopic information for proton-unbound states in ${ }^{23} \mathrm{Mg}$ relevant to the ${ }^{22} \mathrm{Na}(\mathrm{p}, \gamma)$ reaction rate obtained in the present work, including spins/parities and the energy and angular distribution of de-exciting gamma rays. The resonance strengths are extracted in the manner described in the text.

\begin{tabular}{|c|c|c|c|c|c|c|c|c|}
\hline $\begin{array}{c}E_{x} \\
(\mathrm{keV}) \\
\text { Endt } 1998\end{array}$ & $\begin{array}{c}E_{x} \\
\text { Present }\end{array}$ & $\begin{array}{c}E_{\mathrm{p}}(\mathrm{lab}) \\
(\mathrm{keV})\end{array}$ & $I_{i}^{\pi}$ & $I_{f}^{\pi}$ & $\begin{array}{c}E_{\gamma} \\
(\mathrm{keV})\end{array}$ & $\begin{array}{c}\tau \\
(\mathrm{fs})\end{array}$ & $R_{D C O}$ & $\begin{array}{c}\omega \gamma \\
(\mathrm{meV})\end{array}$ \\
\hline $7622(6)$ & $7623.4(9)$ & $45.8(16)$ & $9 / 2^{+}$ & $5 / 2^{+}$ & $7172.5(9)$ & $4(2)$ & $1.57(24)$ & $1.7_{-1.1}^{+2.5} \times 10^{-13}$ \\
\hline $7643(10)$ & $7646.9(26)$ & $73.5(30)$ & $3 / 2^{+}$ & $5 / 2^{+}$ & $7196.0(26)$ & & $0.87(15)$ & $2.2_{-1.4}^{+3.0} \times 10^{-9}$ \\
\hline & $7769.2(10)$ & $198.2(19)$ & $\left(9 / 2^{-}\right)$ & $\begin{array}{c}9 / 2^{+} \\
11 / 2^{+}\end{array}$ & $\begin{array}{l}5054.8(6) \\
2316.9(5)\end{array}$ & $2(1)$ & & $4.0_{-4.0}$ \\
\hline $7783(3)$ & $7779.9(9)$ & 209.4(17) & $11 / 2^{+}$ & $\begin{array}{l}7 / 2^{+} \\
9 / 2^{+}\end{array}$ & $\begin{array}{l}5729.1(11) \\
5067.1(11)\end{array}$ & $<1$ & $1.42(11)$ & $\left(5 \times 10^{-2}\right)$ \\
\hline $7783(3)$ & $7784.6(11)$ & $214.3(18)$ & $7 / 2^{+}$ & $5 / 2^{+}$ & $7333.7(11)$ & $10(3)$ & $0.89(5)$ & $1.8_{-0.7}^{+0.7}$ \\
\hline $7801(2)$ & unobs. & & $5 / 2^{+}$ & & & & & $2.2_{-1.0}^{+1.0}$ \\
\hline $7857(2)$ & $7851.5(14)$ & $284.3(20)$ & $\left(7 / 2^{+}\right)$ & $9 / 2^{+}$ & $5138.1(13)$ & & & $15.8_{-3.4}^{+3.4}$ \\
\hline $8017(2)$ & $8015.3(17)$ & $455.5(23)$ & $\left(5 / 2^{+}-11 / 2^{+}\right)$ & $\begin{array}{l}9 / 2^{+} \\
7 / 2^{+}\end{array}$ & $\begin{array}{c}5300.2(9) \\
5966.7(11)\end{array}$ & & & $68_{-20}^{+20}$ \\
\hline $8166(2)$ & $8159.7(20)$ & $606.5(25)$ & $\left(5 / 2^{+}\right)$ & $7 / 2^{+}$ & $6109.5(18)$ & & & $235_{-33}^{+33}$ \\
\hline
\end{tabular}

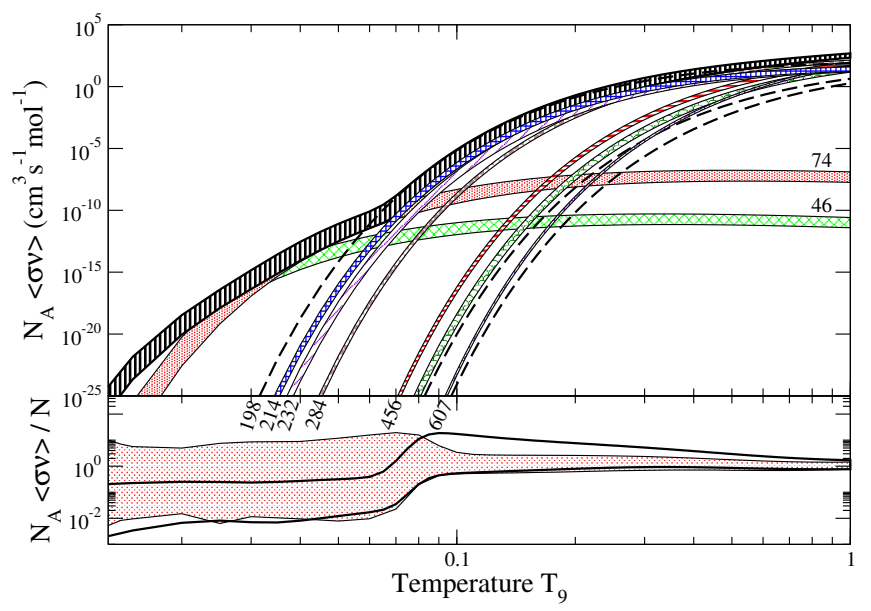

Fig. 2. The top panel presents individual contributions to the total reaction rate labelled by their corresponding proton energy, $E_{\mathrm{p}}(\mathrm{lab})$. The bottom panel compares the bounds on the reaction rate determined in the present work (solid lines) with the uncertainties from the most recent compilation in the literature [14].

where

$$
\omega=\frac{2 J+1}{2\left(2 J_{T}+1\right)} .
$$

In the case where $\Gamma_{p} \ll \Gamma_{\gamma}$, this reduces to

$$
\omega \gamma=\omega \Gamma_{p}
$$

Reaction rates were calculated from the individual resonance strengths and energies. The results of this analysis are presented in fig. 2. The changes to the reaction rate with respect to the compilation in the literature [14] have two principal origins. Firstly, we have fully constrained the spin and parity of the two near-threshold resonances showing that they are associated with $l=2$ rather than $l=0$ and hence, their contribution at lower temperatures will be lower than previously thought. Second, we have identified an additional state in the Gamow window at $E_{\mathrm{p}}(\mathrm{lab})=198 \mathrm{keV}$, which mirror symmetry considerations suggest may have $J^{\pi}=9 / 2^{-}$. If this state did have this spin/parity, then it might, in principle, make a substantial contribution to the overall reaction rate. The contribution is, however, constrained by limited direct measurements made previously. We set an upper limit on the resonance strength for this new state of $4 \mathrm{meV}$. Clearly, it is a priority to determine the resonance strength of this state more precisely and further measurements are warranted.

\subsection{Astrophysical implications}

An analysis of the impact of the new rate for the ${ }^{22} \mathrm{Na}(\mathrm{p}, \gamma)$ reaction on the amount of ${ }^{22} \mathrm{Na}$ ejected during nova outbursts has been performed. An evolutionary sequence for a nova outburst hosting an ONe white dwarf of $1.25 \mathrm{M}_{\odot}$, has been computed by means of a spherically symmetric, hydrodynamic, implicit code, in Lagrangian formulation, extensively used in the modeling of such explosions (see ref. [15] for details). If the resonance strength of the newly discovered state were to tend to its upper limit, then this would represent a reduction by roughly a factor of two in terms of the maximum detectability distance for the $1.275 \mathrm{MeV} \gamma$-ray from ${ }^{22} \mathrm{Na}$. This would have important implications for ongoing satellite-based searches for gamma rays from novae such as the ESA INTEGRAL mission.

\section{Example 2: ${ }^{30} \mathrm{P}(\mathrm{p}, \gamma)$}

We have employed a similar methodology in our approach to improving the experimental situation for states in the Gamow window in ${ }^{31} \mathrm{~S}$ relevant to the ${ }^{30} \mathrm{P}(\mathrm{p}, \gamma)$ reaction. Excited states in ${ }^{31} \mathrm{P}$ and ${ }^{31} \mathrm{~S}$ were produced at the same 


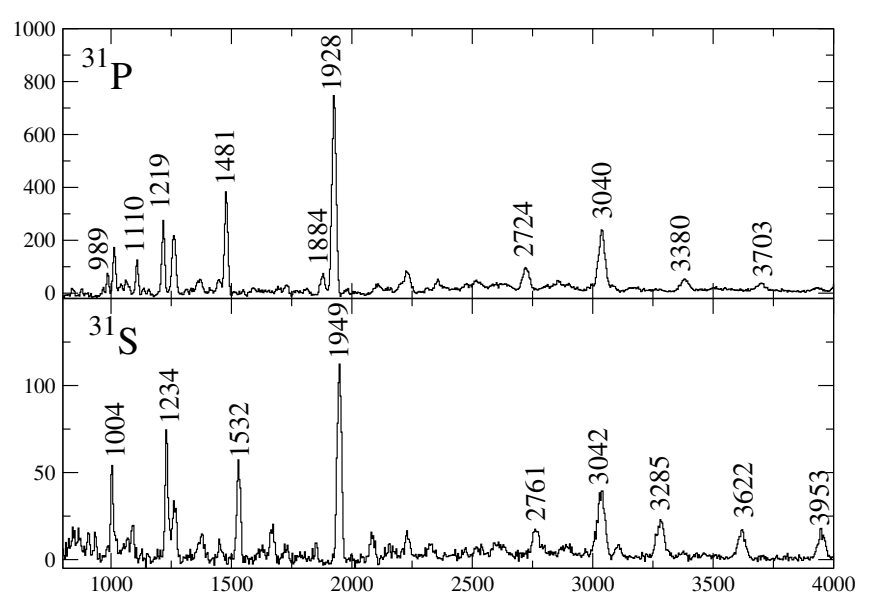

Fig. 3. The top spectrum shows transitions above the $7 / 2^{+}$ level in ${ }^{31} \mathrm{P}$ and is double-gated by the 1266 and $2148 \mathrm{keV}$ transitions in the $\gamma-\gamma-\gamma$ cube. The bottom spectrum contains transitions above the $7 / 2^{+}$level in ${ }^{31} \mathrm{~S}$ and is double-gated by the 1249 and $2102 \mathrm{keV}$ transitions in the $\gamma-\gamma-\gamma$ cube. Mirror transitions of interest are labelled with their energies in $\mathrm{keV}$.

time through the ${ }^{12} \mathrm{C}\left({ }^{20} \mathrm{Ne}, \mathrm{p}\right)$ and ${ }^{12} \mathrm{C}\left({ }^{20} \mathrm{Ne}, \mathrm{n}\right)$ reactions using a $32 \mathrm{MeV}$ beam from the ATLAS accelerator at Argonne National Laboratory. The resulting gamma decays were detected by Gammasphere [10]. Transitions in ${ }^{31} \mathrm{~S}$ were rigorously identified by selecting ${ }^{31} \mathrm{~S}$ recoils using the Fragment Mass Analyser (FMA) [16] and an ion chamber. This information was used in conjunction with a $\gamma-\gamma$ matrix and a $\gamma-\gamma-\gamma$ cube to develop level schemes for the two nuclei. An angular-correlation analysis was performed for the strongest gamma rays observed.

We were able to very cleanly identify the strongest transitions in ${ }^{31} \mathrm{~S}$ when we demanded that the separated recoils had $A=31$ and $Z=16$. The statistics did not allow, however, the identification of weak or high-energy $\gamma$-rays which might be feeding the ground state, given the very low coincident efficiency. In order to develop the level scheme, we exploited the $\gamma$-ray coincidence data, which did not have a condition on the detection of ${ }^{31} \mathrm{~S}$ residues. The analysis is, therefore, complicated by the presence of transitions with near identical energy in other nuclei which were more strongly populated in the experiment. For example, the first $5 / 2^{+}$state in ${ }^{31} \mathrm{~S}$ decays by a $2236 \mathrm{keV}$ $\gamma$-ray, which is near degenerate with strong transitions in both ${ }^{31} \mathrm{P}$ and ${ }^{30} \mathrm{Si}$, and so it is useless for a $\gamma-\gamma$ analysis. We were able, however, to find clean gating conditions to observe transitions feeding the $3 / 2_{1}^{+}, 5 / 2_{2}^{+}, 7 / 2_{1}^{+}$and $7 / 2-$ levels in ${ }^{31} \mathrm{~S}$. An example of the quality of the data obtained, as well as an illustration of the close mirror symmetry of ${ }^{31} \mathrm{~S}$ and ${ }^{31} \mathrm{P}$ is given in fig. 3 .

A re-evaluation of the ${ }^{30} \mathrm{P}(\mathrm{p}, \gamma)$ reaction rate is presently being prepared for publication. Essentially, we have identified many of the states in the Gamow window in ${ }^{31} \mathrm{~S}$, in particular, those with high spin. These are not directly relevant to the proton capture reaction. However, with these states eliminated from the analysis, it becomes possible to make a one-to-one matching of the remaining levels with their known mirror counterparts in ${ }^{31} \mathrm{P}$.
Taken together with calculations of proton spectroscopic factors for the $s d$ shell model states, this allows many of the uncertainties in the reaction rate to be strongly reduced. A very important feature of this analysis has been the occurrence of striking mirror energy shifts between certain states in ${ }^{31} \mathrm{~S}$ and ${ }^{31} \mathrm{P}$, most notably for particular negative-parity states such as the first $9 / 2^{-}$and $13 / 2^{-}$ levels. These mirror energy shifts are found, in some cases, to exceed $250 \mathrm{keV}$. It is believed that these shifts arise from the effects of the electromagnetic spin orbit interaction and their origin is discussed elsewhere [17]. These mirror energy shifts are by no means universal and appear to occur only for particularly pure stretched configurations - negligible energy shifts are observed between the first $7 / 2^{-}$and $11 / 2^{-}$levels, for example [17]. The understanding of nuclear structure effects such as these mirror energy shifts is therefore very important in determining the ${ }^{30} \mathrm{P}(\mathrm{p}, \gamma)$ reaction rate.

\subsection{Outlook for future measurements}

We have considerably improved the experimental information for unbound states in ${ }^{31} \mathrm{~S}$. From the present measurements and careful comparisons with mirror states, there are no ambiguities on spin/parity assignments for the near-threshold resonances whose proton widths principally determine the reaction rate at lower temperatures. The uncertainties now rest principally in the excitation energy of the unmeasured resonances, which are $10-15 \mathrm{keV}$ as determined in transfer reactions, and the unknown spectroscopic factors for low-spin negative-parity states in the Gamow window. The latter are very difficult to calculate since it would be necessary to have a full $s d$ $p f$ shell model calculation. It would, therefore, be fundamentally difficult to improve on the measurement described here. The best approach would be to measure the ${ }^{30} \mathrm{P}(\mathrm{p}, \gamma)$ reaction directly - in particular for low-spin negative-parity resonances. This would be a challenging measurement given the difficulty in producing a beam of ${ }^{30} \mathrm{P}$ using the ISOL technique, though "in-flight" production via the ${ }^{30} \mathrm{Si}(\mathrm{p}, \mathrm{n})$ reaction may be possible. Given a modest radioactive beam of $\sim 10^{6} \mathrm{~s}^{-1}$, a $\left({ }^{3} \mathrm{He}, \mathrm{d}\right)$ reaction could be performed to measure spectroscopic factors for these states. The inaccurate spectroscopic factors are not, however, the only source of error since the error in the resonance energies must also be considered. The uncertainty in the resonance energies might, in principle, be reduced slightly, given a very careful transfer measurement. The inclusion of germanium detectors might be beneficial in this regard, but depending on the magnitude of the gamma branch, this might not be practical.

\section{Conclusions}

We have used a nuclear structure methodology to revisit the question of the ${ }^{22} \mathrm{Na}(\mathrm{p}, \gamma)$ and ${ }^{30} \mathrm{P}(\mathrm{p}, \gamma)$ reaction rates. The former work is published elsewhere [11], while the latter is being finalised for publication. We have shown that 
the complementary approach described here allows resonance energies to be obtained with high precision and ambiguities on spin/parity assignments to be lifted. Clearly, such a methodology is applicable to many other cases than the examples described here and, with modern $4 \pi$ gammaray detector arrays, it is not difficult to perform very detailed spectroscopic studies on unbound states of $s d$ and $s d-f p$ shell nuclei.

\section{References}

1. J. Jose, A. Coc, M. Hernanz, Astrophys. J. 520, 347 (1999).

2. M. Hernanz, in Classical Nova Explosions, edited by M. Hernanz, J. José (AIP, Melville, New York, 2002) p. 399.

3. S. Bishop, R.E. Azuma, L. Buchmann, A.A. Chen et al., Phys. Rev. Lett. 90, 162501 (2003).

4. D. Seweryniak et al., Phys. Rev. Lett. 94, 032501 (2005).
5. J. Jose, A. Coc, M. Hernanz, Astrophys. J. 560, 897 (2001).

6. S. Seuthe et al., Nucl. Phys. A 514, 471 (1990).

7. F. Stegmüller, C. Rolfs, S. Schmidt, W.H. Schulte, H.P. Trautvetter, R.W. Kavanagh, Nucl. Phys. A 601, 168 (1996).

8. S. Schmidt et al., Nucl. Phys. A 591, 227 (1995).

9. S. Kubono et al., Z. Phys. A 348, 59 (1994).

10. I.Y. Lee, Nucl. Phys. A 520, 641c (1990).

11. D.G. Jenkins et al., Phys. Rev. Lett. 92, 031101 (2004).

12. R.B. Firestone, V.S. Shirley (Editors), Table of Isotopes, Vol. 1 (J. Wiley and Sons, 1996).

13. B. Cederwall et al., Nucl. Instrum. Methods Phys. Res. A 353, 591 (1994).

14. C. Angulo et al., Nucl. Phys. A 656, 3 (1999).

15. J. José, M. Hernanz, Astrophys. J. 494, 680 (1998).

16. C.N. Davids, Nucl. Instrum. Methods Phys. Res. B 70, 358 (1992).

17. D.G. Jenkins et al., Phys. Rev. C 72, 031303 (2005). 